\title{
A New Protocol for Controlled Ovarian Stimulation Monitoring by Self-Determination of Estrone-3-Glucuronide and Single Ultrasound (COSSESU)
}

\author{
Iavor K. Vladimirov ${ }^{1,2 *}$, Martin Vladimirov', Desislava Tacheva ${ }^{1,2}$ \\ ${ }^{1}$ IVF Unit, SBALAGRM-Sofia, Sofia, Bulgaria \\ ${ }^{2}$ Faculty of Biology, Sofia University "St. Kliment Ohridski”, Sofia, Bulgaria \\ Email: *vladimirov@ivf.bg, martin.vladimirov@ivf.bg, tacheva@ivf.bg
}

How to cite this paper: Vladimirov, I.K., Vladimirov, M. and Tacheva, D. (2021) A New Protocol for Controlled Ovarian Stimulation Monitoring by Self-Determination of Estrone-3-Glucuronide and Single Ultrasound (COSSESU). Open Journal of Obstetrics and Gynecology, 11, 1217-1228. https://doi.org/10.4236/ojog.2021.119115

Received: August 27, 2021

Accepted: September 23, 2021

Published: September 26, 2021

Copyright $\odot 2021$ by author(s) and Scientific Research Publishing Inc. This work is licensed under the Creative Commons Attribution International License (CC BY 4.0).

http://creativecommons.org/licenses/by/4.0/

\begin{abstract}
Background: Answering the question of what is the optimal protocol for monitoring controlled ovarian stimulation (COS) still remains a challenge. The rapid introduction of new diagnostic methods and various components of telemedicine makes it possible to reduce the number of patient visits during ovarian stimulation, which will reduce the loss of time, costs, and risk for the patient from COVID-like situations. Methods: The different COS monitoring protocols are examined, thus proposing a new approach consisting of two successive phases. Results: In the first phase, E3G in urine samples is being examined, which is performed by the patient themselves with a small portable analyzer. Based on the results, the specialist prescribes the doses for stimulation. The second phase involves one single determination of the size and number of follicles at the end of stimulation, using TVUS, as well as the dynamics of serum levels of $\mathrm{P} 4$ and E2. This proves to be in many cases sufficient. Conclusions: It is of our opinion that on the basis of new diagnostic tests such as E3G in urine and telemedicine, patients are able to independently and actively participate in the treatment process. This new approach to COS monitoring can be successfully implemented in different protocols for ovarian stimulation.
\end{abstract}

\section{Keywords}

COS Monitoring, E3G, Estradiol, TVUS, Telemedicine

\section{Introduction}

Any innovation in assisted reproduction aims to increase the success rate of the 
method and to decrease the risks and costs for the patients. For over 40 years, the success rate of in vitro technologies has increased many times: in the first couple of years with less than $10 \%$, currently more than $50 \%$ in a specific group of patients. The reason for such a rapid increase is the improvement in lab conditions and cultivation media, the application of newer methods and technologies, like PGD/PGS, vitrification of reproductive cells and tissue, determination of an implantation "window", etc. Last, but not least, the success of IVF technologies is also due to the implementation of new medicine and stimulation protocols of the ovary in order to aspirate oocytes of optimal quality and quantity and to reduce risk of serious complications, such as ovarian hyper stimulation syndrome (OHSS).

\section{Monitoring of Controlled Ovarian Stimulation (COS)}

A substantial part of the stimulation protocol is the monitoring of follicle, hormone, and endometrium growth during gonadotropin application. The phrase "monitoring" refers to "close, ongoing surveillance." The goal of monitoring is to assist the physician in selecting the most appropriate protocol or modifying the dose and/or method for the protocol currently being used, in order to achieve the best possible outcome and avoid therapy or procedure-related problems. Transvaginal ultrasound (TVUS) measurements of follicle growth, combined with close monitoring of circulating reproductive hormones, including estradiol, LH, and progesterone levels, are the standard basic monitoring tools in most IVF clinics for controlled ovarian stimulation (COS) during IVF procedure (Figure 1).

A question arises: what is the place and usefulness of the stimulation protocol in every one of the listed examinations and tests? Confino [1] et al. determine that an efficacy of $88 \%$ was observed in GnRH-agonist protocol, with separate TVUS measurement of follicular growth, in comparison to combined TVUS with estradiol (E2), luteinizing hormone (LH), and progesterone (P4). The follicle sonography does not succeed in foreseeing inadequate E2 levels in $8 \%$ of

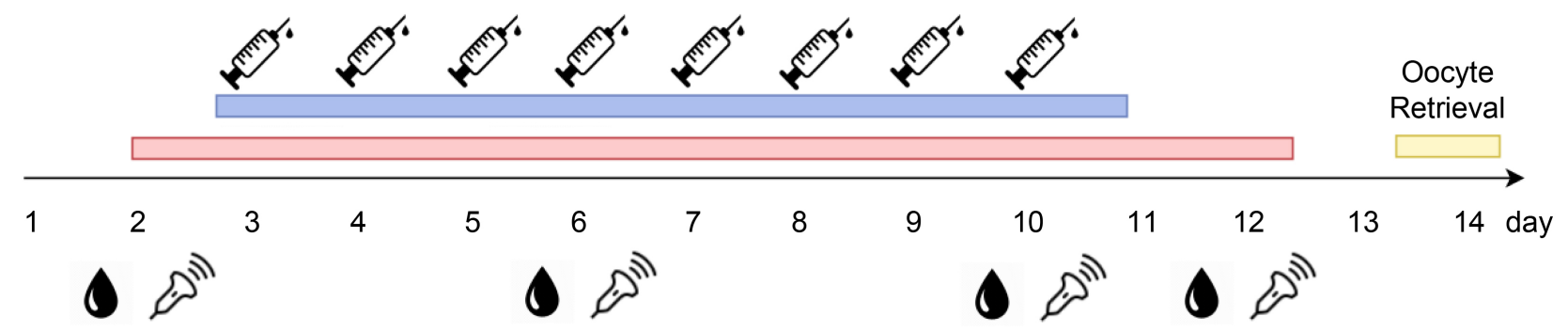

( - Serum E2, P4, LH

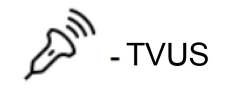

Eే - Gonadotropin Injections

Figure 1. A standard protocol of controlled ovarian stimulation (COS) monitoring. 
cases, while in $3 \%$ of patients, a preliminary LH increase was observed. According to other authors, the inclusion of examining changing E2 levels with the ultrasound exam during stimulation radically changes the ovulation trigger and does not increase the percentage of pregnancies or risk of ovarian hyper stimulation syndrome (OHSS). This applies to the so-called norm group of patients, using down-regulation protocol [2].

Analyzing six randomized controlled trials (RCTs), examining 1515 cases, the authors summarize that $\mathrm{COS}$ monitoring only by ultrasound has a small chance to have a significant increase of clinical pregnancy rates. The number of obtained oocytes was similar to that of combined monitoring with ultrasound and hormonal tests [3].

Another article concludes that the results above should be analyzed with increased caution, as the quality of evidence is very low for all comparisons. The limitations include inaccuracies due to the small group of patients, important clinical results were not taken into consideration, not all patient data was sampled, examination methods were poorly described, and/or clinical heterogeneity was absent. The analysis concludes that, at least in women with an increased risk of OHSS, a combined monitoring protocol should remain, due to the low quality and quantity of contemporary data [4].

Analyzing the discussions, it can be concluded that the optimal monitoring of $\mathrm{COS}$ is still being studied. The search for new methods and strategies to optimize COS monitoring, including elements of telemedicine, continues.

\section{Telemedicine and COS Monitoring}

During the last couple of years, a new medical technology has garnered interest: telemedicine, healthcare provided by telecommunication means [5]. Although much is being published regarding telemedicine in the fields of cardiology, diabetics, dermatology, and common practice, not much is being researched in reproductive medicine [6] [7]. For obvious reasons, interest has gone up in a very short period of time due to the recent COVID-19 pandemic. Telemedicine has a place in fertility treatment through the means of in vitro technologies. This can decrease the cost of treating patients. Pharmaceutical and procedure costs are well-known and can be accounted for in the budget. The side costs aren't few, however, and can prove difficult to foresee. The direct non-medical costs and indirect costs of IVF treatment represent $\sim 45 \%-52 \%$ of the total cost [8]. A study by $\mathrm{Wu}$ et al. [9] shows that only the time costs of performing the whole procedure is 162 hours. Another study [10] proves that, depending on the distance to the clinic, patients can spend anywhere between 15 to 75 hours for trips, with the main costs being food and accommodation, in the range of $104-703$ Euro.

A successful example of IVF telemedicine is the monitoring of the ovarian response by Gerris, et al. [11], which created a new method of self-operated endovaginal telemonitoring (SOET), enabling patients to perform vaginal sonogra- 
phies from the comfort of their own homes. This gives the patient the option not to come to the clinic as often. Applying telemedicine elements through SOET in COS monitoring during IVF treatment saves the patients and their partners time and costs. Structured communication per email or other means allows the patient to be treated in a clinic of their choice, due to the remote nature of the procedure. This increases patient autonomy.

\section{COSSESU: A New Approach}

The combined COS monitoring protocol suggested by us, the so-called Controlled ovarian stimulation by self-determination of estrone-3-glucuronide and single ultrasound (COSSESU), has the same objectives. It includes two main components. The first one is based on determining E3G levels in urine by the patient themselves, from their own homes. It is well-known that an increase of E3G levels correlates with increase in E2 and follicle growth during COS [12]. The goal of determining E3G/E2 levels is to establish follicle growth dynamics and to observe the ovarian response. On the basis of those results, gonadotrophin dosage will be regulated, respectively: either increased, decreased, or unchanged [13]. The second component, which is in the end of the stimulation, includes a single TVUS examination of follicle and endometrium growth, as well as a single determination of $\mathrm{E} 2$ and $\mathrm{P} 4$ levels.

In COSSESU, some telemedicine elements are being used, like self-examination of E3G levels in urine samples. This is done by the patient themselves with a small portable analyzer. The results are sent to the clinic for analysis. Instructions concerning the correct dosage follow. In the patient survey, only 3 out of 34 interviewed couples shared concerns of whether they will manage to determine E3G levels by themselves. Couples shared that the main benefits are saving time, money, decreased risk of COVID-19 infection, and mitigating the stress caused by the often performed venipunctures.

The method is based on a different strategy and philosophy of COS monitoring. It is established that during the first 7 - 10 days of gonadotropin stimulation, indirect methods for evaluating the follicle growth dynamic, without the usage of TVUS technology, can be used. It is well-known that a dynamic change in serum E2 levels is present during COS, which directly correlates with follicle growth [14] [15] [16]. Determining E2 on its own can be used as a marker for follicle growth during stimulation and a single TVUS examination in the end is enough to determine the ovulation trigger [17] [18]. In practice, however, E2 examinations are often combined with the regular ultrasound examinations in clinics. Also, serum E2 requires qualified medical personnel for venipuncture, as well as laboratory and medical analysis of the results. On the other hand, no matter that serum E2 levels correlate with follicle growth, the objective information regarding follicle growth and numbers, obtained by a TVUS examination especially at the end of the stimulation, cannot be replaced. For this reason, it is not recommended to base the timing of the final oocyte maturation trigger on estradiol le- 
vels alone [19].

Analyzing the information stated above, the question arises, how can the patients' "drawbacks" be mitigated, especially when it comes to additional costs of the IVF procedure, spent time, and extra stress of waiting times, venipuncture and other medical procedures? Is there a method or a way to let patients perform some examinations on their own, without the doctor letting go of the stimulation control? In which COS phase is TVUS diagnostics required, supported by the additional information of serum E2 and progesterone levels? It is of our strong belief that the COSSESU protocol is adequate for this goal.

\section{Characteristics}

This protocol will be split into two parts. The first phase begins in day 2 - 3 of the menstrual cycle, with regular examination of urine E3G levels and injection of predetermined doses of gonadotropins, in correlation with the test results. $\mathrm{Pa}$ tients are provided with a small, safe, and easy-to-use in-home monitor customized device, consisting of a fluorescent immunoassay Mira Fertility Plus ${ }^{\circledR}$ (an FDA-approved and CE-marked) analyzer. The device contains individual single-use tests and an instructional leaflet. Patients are not required to undergo special training beforehand. An easy usage algorithm is applied: in the morning, during the patient's first restroom use, the patient deposits a urine sample in a cup, at least 6 - 8 hours after the patient's last restroom use. The patient should then dip the test wand into urine for 10 seconds. The wand is inserted into the Mira Analyzer and the patient has to wait approximately 16 minutes to obtain the E3G results. They are sent securely over the internet or by phone to the center where they are analyzed and stored. A few hours later a structured response is sent informing the patient about the dose of gonadotrophins to be self-injected the same day and/or following day(s). The timing of the next urine E3G analysis will be in the next day or after the next day, depending on the phase of stimulation or if more information is required for the stimulation to continue. E3G urine analysis is recommended to be performed every day, in order to obtain more objective information and to minimize error risks (Figure 2).

The second phase requires a single ultrasound endometrium examination, which is sufficient to determine future treatment [19]. The ultrasound examination also provides information on the number and size of growing follicles and a view of the lesser pelvis, which is important for the following follicular puncture and to search for medical findings (such as hydrosalpinx) that should be taken into consideration during the future embryo transfer. Depending on the stimulation protocol, measurements of serum E2 can also be undertaken, which aim to determine the day and type of ovulation trigger and OHSS risk. Serum progesterone levels can also be measured, in order to determine if fresh or thawed embryo transfer is required in the future.

\section{COSSESU's Advantages}

What are the arguments in favor of COSSESU? Can this method be used 
Controlled ovarian stimulation by self-determination of estrone-3-glucuronide and single ultrasound (COSSESU)

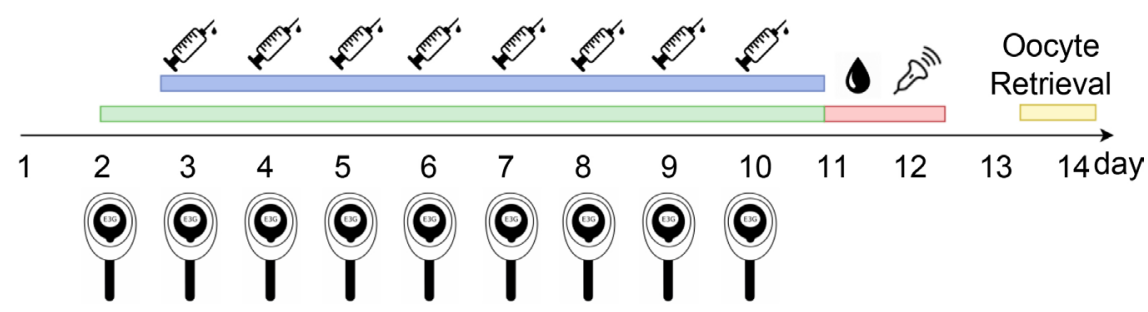

(ख) -Urine E3G -Serum E2, P4 D" -TVUS - Gonadotropin Self-Injections

Figure 2. Day-by-day application of the controlled ovarian stimulation by self-determination of estrone-3-glucuronide and single ultrasound (COSSESU) protocol.

successfully? How objective is it during COS?

A couple of new methods and technologies, which determine the realization of this method, are in use:

- Ovarian reserve assessment, where AMH and the number of antral follicles are objective markers for determining the starting dosage during stimulation [20].

- Gonadotropin injection from the patient themselves. The pharmaceutical industry's contemporary technologies have reached a level that allows the self-injection of the stimulation medicine. This does not require the input of medical personnel, and the dosage can be managed by the doctor online.

- Reliable protocols for stimulation. COS protocols can be individualized, as the ovarian reserve and the patient's age have to be taken into consideration. The GnRH-antagonist protocol is best suited for cases with increased OHSS risk, as the application of a GnRH-agonist as an ovulational trigger significantly decreases the risk of a serious complication as a result of the stimulation. Progestin-primed ovarian stimulation (PPOS) has recently been implemented as an effective alternative to conventional protocols for preventing premature LH-spike, allowing similar results to be obtained. Progestin administration in the follicular phase has been successfully used in normally ovulating patients [21], those with polycystic ovary syndrome (Wang, et al., 2016), and those with a poor response [22]. Progestins can present an effective option for women who do not contemplate fresh ET, e.g. cases with risk of hyper response, preimplantation genetic testing, oocyte donors, or fertility preservation [23].

Different stimulation protocols have different features. The quantity and size of developing follicles, as well as the level of oestradiol, influence the flexibility of GnRH-antagonist use [24] [25]. The first application of a GnRH-antagonist is often in the 6-7th day of the menstrual cycle. One of its basic indications are the 
E2 levels. The wide range between $300-1100 \mathrm{pg} / \mathrm{ml}$ allows better flexibility in GnRH-antagonist application and does not have a negative effect on follicle growth or the quality of the aspirated oocytes [26] [27]. In GnRH-agonist and Progestin-primed ovarian stimulation protocols, managing these has become easier, as the stimulation dosage has to be in-line with the E2 levels in the different days of stimulation, as well as hormone level growth. There is an insignificantly small risk of LH-levels rising, which makes the management of the stimulation much easier.

Research shows that there is a tight correlation between E3G and E2 dynamics in the menstrual cycle and during ovulation induction [28] [29]. The rising E2 serum levels during COS correlate tightly with the rising E3G metabolites in urine [12]. This provides the patient with the opportunity to examine from their own home the changing steroid levels during stimulation, thanks to a portable and compact immunofluorescence analyzer. The results can be easily transferred to the clinic online.

\section{Comparison between COSSESU and SOET Methods}

One of the main advantages of the first phase of the COSSESU protocol is the active participation of the patient through self-determination of E3G in the urine and the use of the opportunities provided by modern telemedicine. Another advantage is that, in the second phase of stimulation, TVUS is performed by a specialist in the clinic, where the serum levels of reproductive hormones are measured, as well. However, both phases of COSSESU alone would not be effective for the optimal monitoring of ovarian stimulation.

SOET's main advantage over COSSESU is the full autonomy of the patient until their first visit in the clinic for follicle puncture. Patients perform sonograms at home, or wherever they happen to be, as long as they have good WiFi access. However, SOET also has a set of drawbacks: First and foremost, patient triage must be undertaken. Not every patient can use a TVUS probe by their own. Using an educated guess, $50 \%$ of the population could be SOET candidates (Gerries, et al., 2009) [11]. Sometimes, there is a psychological barrier that prevents help from the partner. On the other hand, patients sometimes do not perform well even after training. Also, in Gerries et al.'s randomized controlled trial (2014), due to various reasons, one third of the participants were disqualified [30].

Not determining P4 levels before triggering ovulation suggests a Freeze All policy for the embryos, because there is not enough information whether there is an early rise in P4, which decreases success rate in fresh ET [19]. It is well-known that in more than one third of cases, mainly in antagonist stimulation protocol, there is an early rise in P4 [31]. Evidence shows that implantation rates are lower after fresh embryo transfer [32]. Early administration of the trigger for ovulation at the end of COS, based only on the result of TVUS, at low levels of P4, also has a negative effect on the quality of oocytes and the possibility of high pregnancy 
rate [33] [34].

COSSESU circumvents this issue. TVUS performed by a specialist has a list of benefits and is applicable for every single patient. No matter the patient's preliminary training in SOET, it cannot be compared to the experience of an ultrasound specialist, who could discover medical finds during the examination (hydrosalpinx, polyps, myoma, etc.). All of these have a negative effect on the next steps in the treatment by in vitro procedure. A visit to the clinic and a consultation with a specialist also have an important psychological benefit: the patient has to make sure that the stimulation has been successful, the hormonal balance is in order, and that there are no incurred risks with neither OHSS, nor the procedure itself, and that respectively the end result has been obtained.

COSSESU's disadvantage is that, due to the faster follicle and E3G growth in urine, the patient may need to visit the clinic sooner to perform TVUS and to examine serum E2 and P4 levels. Sometimes E3G growth and follicle growth dynamics do not correlate. This could be due to a shorter period of time between the evening and morning urine deposit. This may cause E3G concentration to be lower in urine, which will diminish objectivity. Another drawback in this method is that E3G is not being measured for 24-hour urine samples and there is a risk of variability in results. Here a basic advantage of COSSESU can be used: urine E3G levels can be examined every day, or even a couple of times a day, if necessary. The results can be immediately obtained in the clinic and can be analyzed by a professional, followed by a prescription of stimulation dosage.

\section{Comparison with Other Methods}

Another alternative in COSSESU to urine E3G use with the goal of COS monitoring, is measuring E2 saliva levels. A number of studies have shown that a substantial correlation was discovered between serum and salivary E2 concentrations [35] [36]. Furthermore, the number of follicles and their average diameter, as determined by TVUS, is substantially correlated with both. However, when determining salivary E2 levels, compared to urinary E3G levels, there are a number of inconveniences and shortcomings. Samples were always collected more than 30 minutes after consumption of a meal or beverage or brushing of the teeth, where the patients' mouths were rinsed with water 10 minutes before collection. The amount of blood contamination was not quantified, although the collection methods were designed to avoid it. On the other hand, E3G analysis requires physiologically obtained morning urine. The E3G examination is done at home by the patient themselves, by following easy to understand instructions. Also, estradiol salivary levels, on the other hand, must be analyzed in the lab. The transport and lab examination that follow require time and lengthen the chain reaching the doctor, who must process the results and determine the correct stimulation doses. If the patient lives further away or the transport is late, the examination results will be in place a day or two after the probe collection. The method of examining E2 levels in saliva makes the process slower and more 
difficult, which could interfere with the ovarian stimulation monitoring and thus with the doctor's prescription of the gonadotropin stimulation doses in COS.

\section{Conclusions}

COSSESU and SOET are the first successful COS methods to include telemedicine elements.

COSSESU has many advantages over SOET: it can be applied to all patients without having to train them beforehand; SOET can be applied in about $50 \%$ of cases. Another advantage of COSSESU is the protocol's second phase, TVUS, and examining P4 and E2, if necessary. Some could call this a drawback compared to SOET, because COSSESU forces the patient to visit the clinic once, where venipuncture will be performed to examine the hormone levels in serum. From a telemedicine point of view, this is a drawback, but it is difficult to argue against the added objectivity from an ultrasound examination performed by a specialist, or the other advantages described above when examining P4 and E2 levels in the end of COS.

It is in the interest of the patient, the clinic, and society as a whole to mitigate the direct and indirect costs of IVF treatment, but this should not have an effect on the service quality or the end result, i.e. pregnancy and birth. It is an indisputable fact that TVUS monitoring by a specialist in COS is an advantage and that determining serum hormone levels allows IVF treatment success rates to improve.

It is of our belief that COSSESU is a balanced protocol of COS monitoring, whose application is based upon the different tests, relative to the ovarian response, and their optimal objectivity in the different COS phases. At the start and middle of stimulation, the information obtained by TVUS could be replaced by the dynamic of E2, respectively E3G in urine. Telemedicine elements would also be in place, as the patient would evaluate their own E3G levels in urine by themselves, through the means of an easy method and a non-expensive analyzer that can be either bought or rented. This new approach to COS monitoring ends with a one-time determination of the size and number of follicles at the end of stimulation through TVUS by a specialist, as well as the dynamics of serum levels of P4 and E2, which in many cases is sufficient.

\section{Human Rights Statements and Informed Consent}

This article does not contain any studies with human and animal subjects performed by any of the authors.

\section{Clinical Trials Registration}

This study does not include clinical trials.

\section{Conflicts of Interest}

The author declares no conflicts of interest. 


\section{References}

[1] Confino, E., Binor, Z., Molo, M.W., Rawlins, R., Balos, R., Mullaney, K. and Radwanska, E. (1996) Sonographically Monitored Ovarian Stimulation for Assisted Reproduction: A Prospective, Blind Study. Journal of Reproductive Medicine, 41, 7-10.

[2] Lass, A. (2003) Monitoring of in Vitro Fertilization-Embryo Transfer Cycles by Ultrasound versus by Ultrasound and Hormonal Levels: A Prospective, Multicenter, Randomized Study. Fertility and Sterility, 80, 80-85. https://doi.org/10.1016/S0015-0282(03)00558-2

[3] Martins, W.P., Vieira, C.V.R., Teixeira, D.M., Barbosa, M.A.P., Dassunção, L.A. and Nastri, C.O. (2014) Ultrasound for Monitoring Controlled Ovarian Stimulation: A Systematic Review and Meta-Analysis of Randomized Controlled Trials. Ultrasound in Obstetrics \& Gynecology, 43, 25-33. https://doi.org/10.1002/uog.12566

[4] Farquhar, C., Marjoribanks, J., Brown, J., Fauser, B., Lethaby, A., Mourad, S., Rebar, R., Showell, M. and van der Poel, S. (2017) Management of Ovarian Stimulation for IVF: Narrative Review of Evidence Provided for World Health Organization Guidance. Reproductive Biomedicine Online, 35, 3-16. https://doi.org/10.1016/j.rbmo.2017.03.024

[5] Dorsey, E.R. and Topol, E.J. (2016) State of Telehealth. The New England Journal of Medicine, 375, 154-161. https://doi.org/10.1056/NEJMra1601705

[6] Rashid, L., Bashshur, R.L., Shannon, G.W., Smith, B.R. and Woodward, M.A. (2015) The Empirical Evidence for the Telemedicine Intervention in Diabetes Management. Telemedicine Journal and e-Health, 21, 321-354. https://doi.org/10.1089/tmj.2015.0029

[7] Ferreira, A.C., Mahony, O., Hélio, E., Araujo, A., da Silva Costa, E. and Teleultrasound, F. (2015) Historical Perspective and Clinical Application. International Journal of Telemedicine and Applications, 2015, Article ID: 306259. https://doi.org/10.1155/2015/306259

[8] Le, K.D., Vuong, L.N., Ho, T.M., Dang, V.Q., Pham, T.D., Pham, C.T., Norman, R.J. and Mol, B. (2018) A Cost-Effectiveness Analysis of Freeze-Only or Fresh Embryo Transfer in IVF of Non-PCOS Women. Human Reproduction, 33, 1907-1914. https://doi.org/10.1093/humrep/dey253

[9] Wu, A.K., Elliott, P., Katz, P.P. and Smith, J.F. (2013) Time Costs of Fertility Care: The Hidden Hardship of Building a Family. Fertility and Sterility, 99, 2025-2030. https://doi.org/10.1016/j.fertnstert.2013.01.145

[10] Kelly, J., Hughes, C.M. and Harrison, R.F. (2006) The Hidden Costs of IVF. Irish Medical Journal, 99, 142-143.

[11] Gerris, J., Geril, A. and De Sutter, P. (2009) Patient Acceptance of Self-Operated Endovaginal Telemonitoring (SOET): Proof of Concept. Facts, Views and Vision in Obstetrics and Gynaecology, 1, 161-170.

[12] Vladimirov, I., Martin, V. and Desislava, T. (2021) P-670: Urine Estrone-3-Glucuronide (E3G) Assay: Is There Any Place during Ovarian Stimulation for IVF Cycles? $\mathrm{Hu}-$ man Reproduction, 36, i1-i544. https://doi.org/10.1093/humrep/deab130.669

[13] Fatemi, H., Bilger, W., Denis, D., Griesinger, G., La Marca, A., Longobardi, S., et al. (2021) Dose Adjustment of Follicle-Stimulating Hormone (FSH) during Ovarian Stimulation as Part of Medically-Assisted Reproduction in Clinical Studies: A Systematic Review Covering 10 Years (2007-2017). Reproductive Biology and Endocrinology, 19, Article No. 68.

[14] Vargyas, J.M., Marrs, R.P., Kletzky, O.A. and Mishell, D.R. (1982) Correlation of Ultrasonic Measurement of Ovarian Follicle Size and Serum Estradiol Levels in Ovulatory Patients Following Clomiphene Citrate for in Vitro Fertilization. Ameri- 
can Journal of Obstetrics \& Gynecology, 144, 569-573.

https://doi.org/10.1016/0002-9378(82)90229-0

[15] Suchanek, E., Huderera, K., Dobeca, D. and Grizelj, V. (1994) Number of Follicles, Oocytes and Embryos in Human in Vitro Fertilization Is Relative to Serum Estradiol and Progesterone Patterns during Different Types of Ovarian Hyperstimulation. European Journal of Obstetrics \& Gynecology and Reproductive Biology, 56, 121-127. https://doi.org/10.1016/0028-2243(94)90268-2

[16] Hull, M.E., Moghissi, K.S., Magyar, D.M., Hayes, M.F. and Zado, I. (1986) Correlation of Serum Estradiol Levels and Ultrasound Monitoring to Assess Follicular Maturation. Fertility and Sterility, 46, 42-46. https://doi.org/10.1016/S0015-0282(16)49455-0

[17] Roest, J., Verhoeff, A., van Heusden, A.M. and Zeilmaker, G.H. (1995) Minimal Monitoring of Ovarian Hyperstimulation: A Useful Simplification of the Clinical Phase of in Vitro Fertilization Treatment. Fertility and Sterility, 64, 552-556. https://doi.org/10.1016/S0015-0282(16)57791-7

[18] Wikland, M. and Hillensjo (2001) Monitoring IVF Cycles. In: Gardner, D., Wiessman, A., Howles, C. and Shoham, Z., Eds., Textbook of Assisted Reproductive Technologies: Laboratory and Clinical Perspectives, Martin Dinutz Publisher, Boca Raton, 501-504.

[19] ESHRE (2019) Ovarian Stimulation for IVF/ICSI. Reproductive Endocrinology Guideline. https://www.eshre.eu/Guidelines-and-Legal

[20] Broer, S.L., van Disseldorp, J., Broeze, K.A., Dolleman, M., Opmeer, B.C., Bossuyt, P., Eijkemans, M.J., Mol, B.W. and Broekmans, F.J. (2013) Added Value of Ovarian Reserve Testing on Patient Characteristics in the Prediction of Ovarian Response and Ongoing Pregnancy: An Individual Patient Data Approach. Human Reproduction Update, 19, 26-36. https://doi.org/10.1093/humupd/dms041

[21] Zhu, X., Ye, H. and Fu, Y. (2017) Use of Utrogestan during Controlled Ovarian Hyperstimulation in Normally Ovulating Women Undergoing in Vitro Fertilization or Intracytoplasmic Sperm Injection Treatments in Combination with a "Freeze All” Strategy: A Randomized Controlled Dose-Finding Study of 100 mg versus 200 mg. Fertility and Sterility, 107, 379-386.e4. https://doi.org/10.1016/j.fertnstert.2016.10.030

[22] Chen, Q., Wang, Y., Sun, L., Zhang, S., Chai, W., Hong, Q., et al. (2017) Controlled Ovulation of the Dominant Follicle Using Progestin in Minimal Stimulation in Poor Responders. Reproductive Biology and Endocrinology, 15, 1-9.

https://doi.org/10.1186/s12958-017-0291-0

[23] Ata, B., Capuzzo, M., Turkgeldi, E., Yildiz, S. and La Marca, A. (2021) Progestins for Pituitary Suppression during Ovarian Stimulation for ART: A Comprehensive and Systematic Review Including Meta-Analyses. Human Reproduction Update, 27, 48-66. https://doi.org/10.1093/humupd/dmaa040

[24] Kolibianakis, E.M., Venetis, C.A., Kalogeropoulou, L., Papanikolaou, E. and Tarlatzis, B.C. (2011) Fixed versus Flexible Gonadotropin-Releasing Hormone Antagonist Administration in in Vitro Fertilization: A Randomized Controlled Trial. Fertility and Sterility, 95, 558-562. https://doi.org/10.1016/j.fertnstert.2010.05.052

[25] Lainas, T., Zorzovilis, J., Petsas, G., Stavropoulou, G., Cazlaris, H., Daskalak, V., Lainas, G. and Alexopoulou, E. (2005) A Flexible Antagonist Protocol, Earlier, Criteria-Based Initiation of GnRH Antagonist Is Associated with Increased Pregnancy Rates in IVF. Human Reproduction, 20, 2426-2433. https://doi.org/10.1093/humrep/dei106

[26] Brianna, M., Schumacher, L., Mersereau, J.E. and Steiner, A.Z. (2018) Cycle Day, 
Estrogen Level, and Lead Follicle Size: Analysis of 27,790 in Vitro Fertilization Cycles to Determine Optimal Start Criteria for Gonadotropin-Releasing Hormone Antagonist. Fertility and Sterility, 109, 633-637. https://doi.org/10.1016/j.fertnstert.2017.12.021

[27] Olivennes, F., Belaisch-Allart, J., Emperaire, J.C., Dechaud, H., Alvarez, S., Moreau, L., Nicollet, B., Zorn, J.R., Bouchard, P. and Frydman, R. (2000) Prospective, Randomized, Controlled Study of in Vitro Fertilization-Embryo Transfer with a Single Dose of a Luteinizing Hormone-Releasing Hormone (LH-RH) Antagonist (Cetrorelix) or a Depot Formula of an LH-RH Agonist (Triptorelin). Fertility and Sterility, 73, 314-320. https://doi.org/10.1016/S0015-0282(99)00524-5

[28] Honing Jr., R.V., Austin, C.W., Kuzma, D.L., Shapiro, S.S. and Zweibel, W.J. (1982) Ultrasound Evaluation of Estrogen Monitoring for Induction of Ovulation with Menotropins. Fertility and Sterility, 37, 627-632. https://doi.org/10.1016/S0015-0282(16)46273-4

[29] Tanabe, K., Susumu, N., Hand, K., Nishii, K., Ishikawa, I. and Nozawa, S. (2001) Prediction of the Potentially Fertile Period by Urinary Hormone Measurements Using a New Home-Use Monitor: Comparison with Laboratory Hormone Analyses. Human Reproduction, 16, 1619-1624. https://doi.org/10.1093/humrep/16.8.1619

[30] Gerris, J., Annick, D.A., Dhont, N., Frank, V.F., Madoc, B., Magaly, B.M., et al. (2014) Self-Operated Endovaginal Telemonitoring versus Traditional Monitoring of Ovarian Stimulation in Assisted Reproduction: An RCT. Human Reproduction, 29, 1941-1948. https://doi.org/10.1093/humrep/deu168

[31] Elnashar, A. (2010) Progesterone Rise on the Day of HCG Administration (Premature Luteinization) in IVF: An Overdue Update. Journal of Assisted Reproduction and Genetics, 27, 149-155. https://doi.org/10.1007/s10815-010-9393-8

[32] Bosch, E., Labarta, E., Crespo, J., Simon, C., Remohi, J., Jenkins, J., et al. (2010) Circulating Progesterone Levels and Ongoing Pregnancy Rates in Controlled Ovarian Stimulation Cycles for in Vitro Fertilization: Analysis of over 4000 Cycles. Human Reproduction, 25, 2092-2100. https://doi.org/10.1093/humrep/deq125

[33] Vandekerckhove, F., Gerris, J., Vansteelandt, S., De Baerdemaeker, A., Tilleman, K. and De Sutter, P. (2014) Delaying the Oocyte Maturation Trigger by One Day Leads to a Higher Metaphase II Oocyte Yield in IVF/ICSI: A Randomized Controlled Trial. Reproductive Biology and Endocrinology, 12, 31 .

https://doi.org/10.1186/1477-7827-12-31

[34] Santos-Ribeiro, S., Polyzos, N.P., Haentjens, P., Smitz, J., Camus, M., Tournaye, H. and Blockeel, C. (2014) Live Birth Rates after IVF Are Reduced by Both Low and High Progesterone Levels on the Day of Human Chorionic Gonadotrophin Administration. Human Reproduction, 29, 1698-1705.

https://doi.org/10.1093/humrep/deu151

[35] Rottiers, A.S., Dalewyn, L., Somers, S., Alper, M.M., Sakkas, D. and Gerris, J. (2018) Correlation between Sonographic Follow-Up of Follicular Growth, Serum and Salivary Estradiol in Women Undergoing Controlled Ovarian Stimulation (IVF/ICSI). Facts, Views and Vision in Obstetrics and Gynaecology, 10, 173-179.

[36] Sakkas, D., Howles, C.M.L., Atkinson, L., Borini, A., Bosch, E.A., Bryce, C., Cattoli, M., Copperman, A.B., de Bantel, A.F., French, B., Gerris, J., Granger, S.W., Grzegorczyk-Martin, V., Lee, J.A., Levy, M.J., Matin, M.J., Somers, S., Widra, E.A. and Alper, M.M. (2020) A Multi-Centre International Study of Salivary Hormone Oestradiol and Progesterone Measurements in ART Monitoring. Reproductive BioMedicine Online, 42, 421-428. 